\title{
Conhecimento e prática do controle de higiene bucal em pacientes internados em unidades de terapia intensiva
}

Larissa Silva Oliveira*; Ítalo de Macedo Bernardino**; Jéssica Antoniana Lira e Silva***; Rilva Suely Cardoso Castro Lucas****; Sérgio d’Avila****

Cirurgiã-dentista pela Universidade Estadual da Paraíba

** Graduando em Odontologia, Universidade Estadual da Paraíba

Mestranda em Clínica Odontológica, Universidade Estadual da Paraíba

Professor de Saúde Coletiva, Universidade Estadual da Paraíba

\section{RESUMO}

O objetivo deste estudo foi avaliar o conhecimento e as práticas do controle de higiene bucal (HB) em pacientes internados em unidades de terapia intensiva (UTI). Foi realizado um estudo transversal com 41 profissionais de saúde atuantes em UTI por meio da aplicação de questionário composto de dados sociodemográficos e questões sobre a HB de pacientes hospitalizados. Análises estatísticas descritivas e bivariadas, usando o teste exato de Fischer $(p<0,05)$ foram realizadas. A maioria dos profissionais estava na faixa etária de 30 a 39 anos $(n=15 ; 36,6 \%)$, era do sexo feminino $(\mathrm{n}=37 ; 90,2 \%)$ e técnico (a) de Enfermagem $(n=33 ; 80,5 \%)$. A maioria $(\mathrm{n}=38 ; 92,68 \%)$ respondeu que o hospital no qual trabalhava não possui cirurgião-dentista integrado à equipe, o técnico em enfermagem é o profissional responsável pela $\mathrm{HB}$ dos pacientes $(n=41 ; 100 \%)$. Poucos profissionais afirmaram que existia protocolo de $\mathrm{HB}(\mathrm{n}=11$; $26,8 \%$ ) e utilizar gluconato de clorexidina a $0,12 \% \quad(\mathrm{n}=12 ; 29,3 \%)$. Diferença estatisticamente significativa foi observada entre o tipo de hospital e o tipo de substância utilizada para realização da HB ( $p=0,020)$. Evidencia-se a necessidade de desenvolver e ampliar atividades de ensino, pesquisa e extensão relacionadas à Odontologia Hospitalar nos cursos de Odontologia no Brasil.

Descritores: Higiene Bucal. Unidades de Terapia Intensiva. Unidade de Terapia Intensiva. Equipe Hospitalar de Odontologia.

\section{INTRODUÇÃO}

Pacientes hospitalizados frequentemente apresentam saúde debilitada, demandando maior dedicação da equipe profissional $^{1-4}$. O cirurgião-dentista (CD) desempenha importante papel na capacitação da equipe auxiliar hospitalar para a realização de HB eficaz e adequada às particularidades apresentadas pelo paciente. Com o acentuado avanço das investigações na área da saúde, um novo conceito, cada vez mais evidente, fortalece a inter-relação da doença periodontal com doenças sistêmicas ${ }^{5,6}$.

A cavidade bucal tem sido considerada um potente reservatório para os patógenos respiratórios. A falta de adequada $\mathrm{HB}$, nestes 
pacientes, favorece as condições de crescimento bacteriano podendo promover interações bacterianas entre bactérias nativas da placa e patógenos respiratórios, contribuindo, assim, para o desenvolvimento de doenças respiratórias ${ }^{6,7}$. A pneumonia nosocomial é o tipo mais comum, tendo como principal via de ocorrência a aspiração de conteúdos da orofaringe $\mathrm{e}^{8}$.

A implantação dos procedimentos relacionados à Odontologia se faz necessária no contexto hospitalar e sua consolidação implica em infraestrutura que contemple recursos físicos, materiais e intelectuais ${ }^{9,10}$. Frente a esse desafio, é crucial que os cursos articulem atividades de ensino, pesquisa e extensão relacionadas à Odontologia Hospitalar e discutam junto aos graduandos o verdadeiro papel dos profissionais de odontologia no cenário hospitalar, sobretudo nas Unidades de Terapia Intensiva (UTI).

Estudos sobre a avaliação do conhecimento e das práticas do controle de HB em pacientes internados em UTI são essenciais e podem subsidiar a reformulação do cuidado, contribuindo para uma melhor qualidade de vida e bem-estar dos pacientes. Diante deste contexto, o objetivo deste estudo foi avaliar o conhecimento e as práticas do controle de higiene oral em pacientes internados em UTI.

\section{MATERIAL E MÉTODOS}

Foi realizado um estudo transversal com uma abordagem indutiva, por meio de aplicação de questionários direcionados a profissionais de saúde atuantes em UTI de hospitais privados ou públicos/conveniados ao SUS do município de Campina Grande, Paraíba, Brasil. A cidade em questão conta com uma população de aproximadamente 385.213 habitantes, possui uma área total de $594,182 \mathrm{~km}^{2}$ e segundo dados da Secretaria de Saúde, possui 14 hospitais, sendo que destes quatro são públicos e dez privados $^{11}$. O município é dividido em seis distritos sanitários. Em razão do distrito II abranger o maior número de hospitais (nove, sendo seis privados e três públicos), representou o foco deste estudo. Destes, dois foram excluídos por não possuírem UTI e dos sete hospitais restantes, quatro (três públicos e um privado) assinaram o Termo de Autorização Institucional, sendo incluídos neste estudo.

Do universo composto por 60 profissionais de enfermagem (11 enfermeiros e 49 técnicos), 41 concordaram em participar do estudo, assinando o termo de consentimento livre e esclarecido. Previamente à coleta de dados, foi feito um estudo piloto objetivando testar a metodologia proposta. Foram estudadas variáveis relacionadas às características sócio demográficas dos profissionais, ao perfil de formação e ao conhecimento e às práticas de controle de HB de pacientes internados.

Os dados coletados foram, inicialmente, analisados por meio de estatísticas descritivas, que corresponderam ao cálculo de frequências absolutas e percentuais de todas as variáveis investigadas. Por fim, verificou-se a distribuição dos hospitais (privado/público) segundo a existência de protocolo de HB, fornecimento de material para a sua realização e tipo de substância utilizada, assim como foi realizado o teste exato de Fischer a fim de identificar diferenças estatisticamente significativas ao nível de $\mathrm{p}<0,05$. A organização do banco de dados e todas as análises foram feitas usando o software IBM SPSS versão 20.0 e considerando um intervalo de confiança de $95 \%$.

O projeto de pesquisa foi aprovado pelo Comitê de Ética em Pesquisa da Universidade Estadual da Paraíba e foram seguidos todos os preceitos nacionais e internacionais sobre ética em pesquisa envolvendo seres humanos. 


\section{RESULTADOS}

A tabela 1 apresenta a distribuição dos profissionais segundo as características sócio demográficas e relacionadas ao perfil profissional. A maioria estava na faixa etária de 30 a $39 \operatorname{anos}(\mathrm{n}=15 ; 36,6 \%)$, era do sexo feminino ( $\mathrm{n}=37 ; 90,2 \%)$ e técnico (a) de enfermagem ( $\mathrm{n}$ $=33 ; 80,5 \%)$ e trabalhava na área há 11 anos ou mais $(\mathrm{n}=24 ; 58,5 \%)$.

De acordo com a tabela 2, a maioria dos profissionais $(\mathrm{n}=36 ; 87,8 \%)$ trabalha em hospitais públicos/conveniados ao SUS. Um total de $38(92,68 \%)$ respondeu que o hospital no qual trabalhava não possui $\mathrm{CD}$ integrado à equipe. Todos os profissionais $(n=41 ; 100 \%)$ afirmaram que o técnico de enfermagem era o profissional responsável pela $\mathrm{HB}$ dos pacientes. Apenas 11 (26,8\%) afirmaram que havia protocolo de HB. Além disso, 61\% ( $\mathrm{n}=25)$ afirmaram que era fornecido material para a realização da $\mathrm{HB}$. A maioria dos profissionais não utilizava gluconato de clorexidina a $0,12 \%$, mas outras substâncias (cloreto de cetilpri- ridínio e soro fisiológico) para realizar a $\mathrm{HB}$.

Conforme descrito na tabela 3 , na avaliação dos conhecimentos em relação à saúde bucal, 80,5\% (n = 33) afirmaram não ter realizado algum curso ou treinamento para realizar a HB dos pacientes hospitalizados. A necessidade da existência de um protocolo de HB nos hospitais foi reconhecida por $95,1 \%$ (n $=39)$ dos profissionais. Um total de $97,6 \%(\mathrm{n}=$ 40) da equipe de enfermagem destacou que a saúde bucal do paciente é importante para a sua recuperação. A maioria dos profissionais $(\mathrm{n}=$ $32 ; 78,0 \%$ ) considerou importante a presença do CD em ambientes hospitalares.

A tabela 4 mostra a distribuição dos hospitais (privado/público) segundo a existência de protocolo de $\mathrm{HB}$, fornecimento de material para a sua realização e tipo de substância utilizada. O resultado do teste exato de Fischer aponta que existe diferença estatisticamente significativa entre o tipo de hospital e a substância utilizada para realização da HB dos pacientes internados $(p=0,020)$.

Tabela 1 - Características sociodemográficas dos profissionais

\begin{tabular}{|c|c|c|}
\hline Variáveis & $\mathbf{n}$ & $(\%)$ \\
\hline \multicolumn{3}{|l|}{ Idade } \\
\hline 18 a 29 anos & 8 & 19,5 \\
\hline 30 a 39 anos & 15 & 36,6 \\
\hline 40 a 49 anos & 14 & 34,1 \\
\hline 50 a 59 anos & 3 & 7,3 \\
\hline 60 anos ou mais & 1 & 2,4 \\
\hline \multicolumn{3}{|l|}{ Sexo } \\
\hline Feminino & 37 & 90,2 \\
\hline Masculino & 3 & 7,3 \\
\hline Não registrado & 1 & 2,4 \\
\hline \multicolumn{3}{|l|}{ Profissão } \\
\hline Enfermeiro (a) & 8 & 19,5 \\
\hline Técnico (a) de Enfermagem & 33 & 80,5 \\
\hline \multicolumn{3}{|l|}{ Tempo de formação } \\
\hline$\leq 5$ anos & 9 & 22,0 \\
\hline De 6 a 10 anos & 8 & 19,5 \\
\hline$\geq 11$ anos & 24 & 58,5 \\
\hline Total & 41 & 100,0 \\
\hline
\end{tabular}


Tabela 2 - Distribuição dos profissionais segundo o tipo de hospital, a presença de cirurgião-dentista integrado à equipe e a avaliação das práticas de higiene bucal dos pacientes

\begin{tabular}{lcc}
\hline \multicolumn{1}{c}{ Variável } & $\mathbf{n}$ & $\mathbf{\%}$ \\
\hline Tipo de hospital & 5 & 12,2 \\
Privado & 36 & 87,8 \\
Público/Conveniado ao SUS & & \\
Existe um cirurgião-dentista responsável pela saúde bucal dos pacientes? & 0 & 0,0 \\
Sim & 38 & 92,7 \\
Não & 3 & 7,3 \\
Não sei & 0 & 0,0 \\
Quem é o profissional responsável pela higiene bucal dos pacientes em UTI? & 100,0 \\
Enfermeiro (a) & 41 & \\
Técnico (a) & 11 & 26,8 \\
Existe um protocolo de higiene bucal? & 26 & 63,4 \\
Sim & 4 & 9,8 \\
Não & & \\
Não sei & 25 & 61,0 \\
É fornecido material para a sua realização? & 14 & 34,1 \\
Sim & 2 & 4,9 \\
Não & & \\
Não sei & 12 & 29,3 \\
Qual a substância utilizada? & 3 & 7,3 \\
Gluconato de clorexidina a 0,12\% & 8 & 19,5 \\
Triclosan & 17 & 41,5 \\
Nenhum, somente gaze & 9 & 2,4 \\
Outra & & 2,4 \\
Não especificado & 1 & 22,0 \\
Com que frequência se executa a higiene bucal dos pacientes em UTI? & 9 & 61,0 \\
Uma vez ao dia & 25 & 14,6 \\
Duas vezes ao dia & 6 & 100,0 \\
Três vezes ao dia & 41 \\
Outra & & \\
Total & & \\
\hline
\end{tabular}

\section{DISCUSSÃO}

A HB nos hospitais geralmente é atribuída à equipe de enfermagem, destinandose a ela a responsabilidade de garantir o cuidado diário de higiene, inclusive a HB. Este fato também foi observado no presente estudo. Os resultados também evidenciaram a necessidade de se ter um protocolo de higiene oral padronizado. Embora a presença do $\mathrm{CD}$ tenha sido considerada importante pela maioria dos entrevistados, a maioria relatou que esse profissional não se encontra presente nas unidades hospitalares, semelhante ao observado por outros autores na literatura científica ${ }^{5,6}$.
A falta de protocolos padronizados faz com que a $\mathrm{HB}$ dos pacientes seja realizada com menor frequência e os enfermeiros a realizem de acordo com as suas preferências particulares ${ }^{13}$. A maior parte dos profissionais de enfermagem afirmou que o hospital fornece material para realização de $\mathrm{HB}$, corroborando com achados prévios da literatura ${ }^{14}$. É importante considerar que a disponibilidade de materiais e equipamentos para o atendimento ao paciente pode afetar substancialmente a qualidade dos cuidados prestados pelos profissionais da enfermagem.

Quanto à substância utilizada na 
realização da HB nas UTI avaliadas, poucos afirmaram utilizar gluconato de clorexidina a $0,12 \%$.

A clorexidina tem sido considerada como o antisséptico de escolha em protocolos de higiene oral em UTI, representando um potente antimicrobiano, com amplo espectro de ação e alta substantividade ${ }^{15-20}$.

Tabela 3 - Capacitação e percepção dos profissionais sobre a importância da saúde bucal na recuperação do paciente hospitalizado

Variáveis $\quad$ n $(\%)$

\begin{tabular}{lcc}
\hline Você realizou algum curso ou treinamento para realizar a HB dos pacientes hospitalizados? \\
Sim & 7 & 17,1 \\
Não & 33 & 80,5 \\
Não especificado & 1 & 2,4 \\
Você se sente seguro/capaz para exercer tal função? & 33 & 80,5 \\
Sim & 7 & 17,1 \\
Não & 1 & 2,4 \\
Não especificado & 39 & 95,1 \\
Você acha necessária a existência de um protocolo de HB em hospitais? & 1 & 2,4 \\
Sim & 1 & 2,4 \\
Não & \\
Não especificado & 40 & 97,6 \\
Você considera a saúde bucal do paciente importante para a sua recuperação? & 0,0 \\
Sim & 0 & 2,4 \\
Não & 1 & 78,0 \\
Não especificado & 42,0 \\
É importante a integração do cirurgião-dentista à equipe de profissionais atuantes nos hospitais? \\
Sim & 32 & 22,0 \\
Não & 9 & $\mathbf{1 0 0 , 0}$ \\
Total & $\mathbf{4 1}$ \\
\hline
\end{tabular}

Tabela 4 - Distribuição dos hospitais, segundo a existência de protocolo de HB, fornecimento de material para a sua realização e tipo de substância utilizada.

\begin{tabular}{|c|c|c|c|c|c|c|c|}
\hline \multirow{3}{*}{ Variáveis } & \multicolumn{4}{|c|}{ Tipo de Hospital } & \multirow{2}{*}{\multicolumn{2}{|c|}{ Total }} & \multirow{3}{*}{ p-valor } \\
\hline & \multicolumn{2}{|c|}{ Privado } & \multicolumn{2}{|c|}{ Público } & & & \\
\hline & $\mathbf{n}$ & $\%$ & $\mathbf{n}$ & $\%$ & $\mathbf{n}$ & $\%$ & \\
\hline Existência de protocolo de $\mathrm{HB}$ & & & & & & & $0,598^{*}$ \\
\hline Sim & 2 & 18,2 & 9 & 81,8 & 11 & 100,0 & \\
\hline Não & 3 & 10,0 & 27 & 90,0 & 30 & 100,0 & \\
\hline Fornecimento de material para & & & & & & & $0,162 *$ \\
\hline Sim & 5 & 20,0 & 20 & 80,0 & 25 & 100,0 & \\
\hline Não & 0 & 0,0 & 14 & 100,0 & 14 & 100,0 & \\
\hline Não sabe & 0 & 0,0 & 2 & 100,0 & 2 & 100,0 & \\
\hline Substância & & & & & & & $\mathbf{0 , 0 2 0} *$ \\
\hline Gluconato de clorexidina a $0,12 \%$ & 0 & 0,0 & 12 & 100,0 & 12 & 100,0 & \\
\hline Triclosan & 2 & 66,7 & 1 & 33,3 & 3 & 100,0 & \\
\hline Nenhuma, somente gaze & 0 & 0,0 & 8 & 100,0 & 8 & 100,0 & \\
\hline Outra & 3 & 17,6 & 14 & 82,4 & 17 & 100,0 & \\
\hline Não sabe & 0 & 0,0 & 1 & 100,0 & 1 & 100,0 & \\
\hline
\end{tabular}

* Teste exato de Fischer 
Os antissépticos tópicos constituem o método de escolha mais usual na higienização desses pacientes, tendo em vista que, ao contrário dos antibióticos, não há preocupação com o desenvolvimento de resistência microbiana e/ou mudanças na microflora bucal ${ }^{14,21,22}$. Quanto à frequência do controle de infecção bucal em pacientes em UTI, a maior parte dos profissionais afirmou realizar a HB três vezes ao dia. Estudo feito por Grap et al. ${ }^{23}$ identificou que a maioria dos entrevistados assinalou realizar HB duas ou três vezes por dia para pacientes não entubados e 5 vezes por dia ou mais em pacientes entubados.

Quando questionados sobre a participação em algum curso ou treinamento para realização da $\mathrm{HB}$, um expressivo percentual de profissionais afirmou que não. Resultados semelhantes foram reportados por outras pesquisas na literatura ${ }^{5,6,24}$. No entanto, apesar da maior parte dos profissionais não ter realizado curso ou treinamento específico para a realização da $\mathrm{HB}$, poucos relataram se sentir inseguros ou incapazes de realizar tal função. Estudo desenvolvido por Faiçal et al. ${ }^{25}$ identificou que 91,4\% dos auxiliares de enfermagem assumiram necessitar de mais informações sobre cuidados específicos com a saúde bucal.

Muitos profissionais consideraram a HB importante para recuperação do paciente. Araújo et al. ${ }^{5}$ evidenciaram que os profissionais de enfermagem têm conhecimento sobre a relação saúde bucal/saúde geral, sendo que a maioria dos entrevistados concordaram com a afirmativa de que uma infecção na boca pode fazer com que a saúde do resto do corpo seja prejudicada.

Uma das limitações deste estudo referese à recusa imediata de alguns hospitais que possivelmente tiveram receio de expor os métodos de HB utilizados na UTI do estabelecimento ou, até mesmo, por falta de interesse quanto ao assunto proposto. Por outro lado, forneceu informações importantes sobre o conhecimento e práticas de profissionais atuantes nas UTI da região estudada sobre os cuidados com a saúde bucal.

O projeto político-pedagógico do curso de Odontologia deve ser construído coletivamente, centrado no aluno e apoiado no professor como facilitador e mediador do processo de ensino-aprendizagem e articulado entre o ensino, a pesquisa e a extensão, tornando-se essencial verificar a necessidade de ampliação de cenários de aprendizagem durante a graduação ${ }^{26-29}$. Portanto, a realização de estágios supervisionados e o desenvolvimento de projetos de pesquisa e extensão nas UTI poderá contribuir para a integração ensinoserviço, assim como dar espaço para a construção de ricas vivências e experiências para os acadêmicos de Odontologia.

Espera-se também que os resultados obtidos possam subsidiar o planejamento das ações de gestão em saúde, reformulação do cuidado e assistência à saúde bucal. Encoraja-se a integração do $\mathrm{CD}$ à equipe profissional destas instituições, a fim de ampliar as práticas de prevenção e assistência em saúde bucal, contribuindo para uma recuperação mais rápida e melhor qualidade de vida destes pacientes.

\section{CONCLUSÕES}

Os resultados permitem concluir que os cuidados com a saúde bucal dos pacientes nas UTI geralmente é atribuição dos técnicos de enfermagem, os quais na sua grande maioria não recebem orientações específicas para esta atividade e consideram importante a integração do $\mathrm{CD}$ à equipe multiprofissional dos hospitais.

O CD pode contribuir de maneira substancial para a atenção e o cuidado integral dos pacientes nas UTI. A discussão das atribuições e competências do CD no âmbito hospitalar, em 
especial nas UTI, deve iniciar desde a graduação, sendo fundamental a inclusão da Odontologia Hospitalar nos projetos políticopedagógicos dos cursos de Odontologia no Brasil.

\section{ABSTRACT \\ Knowledge and practice of oral hygiene control in hospitalized patients in intensive care units}

The aim of this study was to evaluate the knowledge and practices of oral hygiene $(\mathrm{OH})$ control in patients in intensive care units (ICU). A cross-sectional study was carried out involving 41 health professionals working in ICU by applying a questionnaire composed of sociodemographic data and variables related to the $\mathrm{OH}$ practices. Descriptive and bivariate statistics, using the Fisher's exact test $(\mathrm{p}<0.05)$ were performed. Most of the professionals were aged $30-39$ years $(\mathrm{n}=15 ; 36.6 \%)$, women $(\mathrm{n}=$ $37 ; 90.2 \%)$ and worked as nursing technician (n $=33 ; 80.5 \%)$. Most of them $(\mathrm{n}=38 ; 92.68 \%)$ answered that there is no dentist in the hospitals and that the nursing technician is the professional responsible for the oral hygiene of patients $(n=41 ; 100 \%)$. The existence of oral hygiene protocol $(\mathrm{n}=11 ; 26.8 \%)$ and the use of chlorhexidine gluconate $0.12 \%(\mathrm{n}=12 ; 29.3 \%)$ was unusual. Statistical difference was observed between the hospitals and the substance used for oral hygiene $(\mathrm{p}=0.020)$. It highlights the need to develop and expand the activities of teaching, research and extension related to Hospital Dentistry in Dental courses in Brazil.

Descriptors: Oral Hygiene. Intensive Care Units. Dental Staff, Hospital.

\section{REFERÊNCIAS}

1. Aranega AM, Bassi APF, Ponzoni D, Wayama MT, Esteves JC, Junior IRG. Qual a importância da Odontologia Hospitalar? Rev Bras Odontol. 2012; 69(1):90-3.

2. Feider LL, Mitchell P, Bridges E. Oral care practices for orally intubated critically Ill adults. Am J Crit Care. 2010;19(2):175-83.

3. Martins MTF. Odontologia Hospitalar Intensiva: Interdisciplinaridade e Desafios. Sociedade Mineira de Terapia Intensiva. 2009. [acesso em 02 dez 2012]. Disponível em: http://www.somiti.org.br/pesquisa/ 17_Odontologia\%20nas\%20UTIsok.pdf.

4. Morais TMND, Silva AD, Avi ALRO, Souza PHRD, Knobel E, Camargo LFA. A importância da atuação odontológica em pacientes internados em unidade de terapia intensiva. Rev Bras Ter Intensiva. 2006; 18(4):412-7.

5. Araújo RJGD, Oliveira LCGD, Hanna LMO, Corrêa AM, Carvalho LHV, Alvares NCF. Análise de percepções e ações de cuidados bucais realizados por equipes de enfermagem em unidades de tratamento intensivo. Rev Bras Ter Intensiva. 2009; 21(1):38-44.

6. Araújo RJGD, Vinagre NPL, Sampaio JMS. Avaliação sobre a participação de cirurgiões-dentistas em equipes de assistência ao paciente. Acta Sci Health Sci. 2009;31(2):153-7.

7. Koeman M, Van der ven AJ, Hak E, Joore $\mathrm{HC}$, Kassjager KS, Boten MJ. Oral decontamination with chlorhexidine reduces the incidence of ventilatorassociated pneumonia. Am J Respir Crit Care Med. 2006;173:1348-55.

8. Kahn S, Sardenberg E, Silva LR, Machado WAS, Alves J. Pneumonia por aspiração associada à doença periodontal. Rev Bras Odontol. 2003;60(4):244-6.

9. Miclos PV, Silva Junior MF, Oliveira CMSC, Oliveira MA. Inclusão da Odontologia no cenário hospitalar da região metropolitana de Belo Horizonte, MG. Arq Odontol. 2014; 50(1): 28-34.

10. Pinheiro FMC, Nóbrega-Therrien SM, Almeida MEL, Almeida MI. A formação do cirurgião-dentista no Brasil: contribuições de estudos para a prática da profissão. RGO. 2009; 57(1): 99-106.

11. IBGE - Instituto Brasileiro de Geografia e Estatística. Censo demográfico 2010. [acesso em 01 mai 2013]. Disponível em: 
http://www.ibge.gov.br.

12. Conselho Federal de Enfermagem (Brasil). Lei n. ${ }^{\circ} 7498 / 86$ de 1986 art. 13, regulamentação do exercício de enfermagem e de outras providências. [acesso em 02 dez 2012]. Disponível em: http://www.portalcofen.gov.br.

13. Cutler CJ, Davis N. Improving oral care in patients receiving mechanical ventilation. Am J Crit Care. 2005;14(5) :389-94.

14. Binkley C, Furr LA, Carrico R, McCurren C. Survey of oral care practices in US intensive care units. Am J Infect Control. 2004;32(3):161-9.

15. Deriso AJ, Ladowski JS, Dillonn TA, Justice JW, Peterson AC. Chlorhexidine gluconate $0.12 \%$ oral rinse reduces the incidence of total nosocomial respiratory infection and nonprophylactic systemic antibiotic use in patients undergoing heart surgery. Chest. 1996;109(6):1556-61.

16. Fourrier F, Dubois D, Pronnier P, Herbecq $\mathrm{P}$, Leroy O, Desmettre T, et al. Effect of gingival and dental plaque antiseptic decontamination on nosocomial infections acquired in the intensive care unit: a double-blind placebo-controlled multi center study. Crit Care Med. 2005;33(8) :1728-35.

17. Genuit T, Bochicchio G, Napolitano LM, McCarter RJ, Roghman MC. Prophylactic chlorhexidine oral rinse decreases ventilator-associated pneumonia in surgical ICU patients. Surg Infect. 2001; 2(1):5-18.

18. Herrera BS, Mendes GIAC, Porto RM, Rigato HM, Moreira LD, Muscará MN, et al. O papel da clorexidina no tratamento de pacientes com gengivite no Distrito de São Carlos do Jamari - RO. Periodontia. 2007; 17(4):60-4.

19. Houston S, Hougland P, Anderson JJ, LaRocco M, Kennedy V, Gentry LO. Effectiveness of $0.12 \%$ chlorhexidine gluconate oral rinse in reducing prevalence of nosocomial pneumonia in patients undergoing heart surgery. Am J Crit Care. 2002;11(6):567-70.
20. Marinho BVS, Araújo ACS. O uso dos enxaguatórios bucais sobre a gengivite e o biofilme dental. IJD. 2007;6(4):124-31.

21. Beraldo CC, Andrade D. Higiene bucal com clorexidina na prevenção de pneumonia associada à ventilação mecânica. J Bras Pneumol. 2008; 34(9) :707-14.

22. Bopp M, Darby M, Loftin KC, Broscious S. effects of daily oral care with $0.12 \%$ chlorhexidine gluconate and a standard oral care protocol on the development of nosocomial pneumonia in intubated patients: a pilot study. J Dent Hyg. 2006; 80(3):1-13.

23. Grap MJ, Munro CL, Ashtiani B, Bryant S. Oral care interventions in critical care: frequency and documentation. Am J Crit Care. 2003;12(2):113-8.

24. Chung JP, Mojon P, Budtz-Jorgensen E. Dental care of elderly in nursing homes: perceptions of managers, nurses, and physicians. Spec Care Dent. 2000;20(1):12-7.

25. Faiçal AMB, Mesas AE. Cuidados com a saúde bucal de pacientes hospitalizados: conhecimento e práticas dos auxiliares de enfermagem. Esp Saúde. 2008;10(1):1-6.

26. Fonseca EP. As Diretrizes Curriculares Nacionais e a formação do cirurgiãodentista brasileiro. J Manag Prim Health Care. 2012; 3(2):158-178.

27. Aguiar ASW, Guimarães MV, Morais RMP, Saraiva JLA. Atenção em saúde bucal em nível hospitalar: relato de experiência de integração ensino/serviço em odontologia. Extensio. 2010;7(9):100-110.

28. Godoi APT, Francesco AR, Duarte A, Kemp APT, Silva-Lovato CH. Hospital odontology in Brazil: a general vision. Rev Odontol UNESP. 2009;38(2):105-9.

29. Doro GM, Fialho LM, Losekann M, Pfeiff DN. Projeto "Odontologia Hospitalar". Rev ABENO. 2006;6(1):49-53.

Correspondência para:

Sérgio d'Avila

e-mail: davila2407@hotmail.com

Av. das Baraúnas, 351, Bairro Universitário

58429-500 Campina Grande - PB 